\title{
Colgajo giratorio retroauricular
}

\section{Retroauricular flip-flop flap}

Jorge Jury S. ${ }^{1}$, Catalina Jury S. ${ }^{2}$, Tatiana Sepúlveda S. ${ }^{2}$, Osvaldo Jury S. ${ }^{3}$

\begin{abstract}
Resumen
El colgajo retroauricular en isla (flip-flop flap) fue descrito por Masson en 1972 y consiste en tejido dermoepidérmico irrigado por un pedículo de patrón aleatorio subcutáneo de ramas de la arteria auricular posterior. Proviene de la región mastoidea y retroauricular y aporta buena cobertura para la región anteromedial del pabellón auricular. Se describen dos casos, en que se realizó cobertura inmediata secundario a un defecto de la concha auricular posterior a resección neoplásica, obteniendo resultados satisfactorios y sin complicaciones.
\end{abstract}

Palabras clave: colgajo, puerta giratoria, oreja, reconstrucción, cáncer basocelular.

\begin{abstract}
The retroauricular island flap (flip-flop flap), was described by Masson in 1972 and consists on dermoepidermal tissue irrigated by a random subcutaneous pedicle of branches of the posterior auricular artery. It comes from the mastoid and retroauricular region and provides a good coverage for the anteromedial region of the pinna. Two cases are described, in which immediate coverage was performed secondary to a defect in the auricular concha after a neoplastic resection, obtaining satisfactory results without complications.
\end{abstract}

Keywords: flap, flip-flop, ear, reconstruction, basal cell carcinoma.
${ }^{1}$ Clínica Sanatorio Alemán de Concepción, Chile ${ }^{2}$ Facultad de Medicina, Universidad Católica de la Santísima Concepción. Concepción, Chile.

${ }^{3}$ Facultad de Medicina, Universidad San Sebastián. Concepción, Chile.

Los autores declaran no tener conflictos de interés.

Recibido el 19 de marzo de 2020. Aceptado el 1 de mayo de 2020.

Correspondencia: Jorge Jury $S$. Pedro de Valdivia 801, Concepción, Chile. Email: juryplastic@gmail.com

\section{Introducción}

La concha auricular representa la estructura central del pabellón auricular. Su exacta reconstrucción requiere que cumpla con los requisitos funcionales y estéticos ${ }^{1}$. Una alternativa para la reconstrucción es el colgajo giratorio retroauricular, conocido también como flip-flop flap o en puerta giratoria. Es un colgajo en isla descrito por Masson en 1972, usado para la reconstrucción de defectos del pabellón auricular, especialmente del área de la concha, y cuando se estima que otras alternativas no son suficientes para lograr una cobertura satisfactoria ni estética ${ }^{2}$. La zona donante corresponde a la región posterior de la oreja junto con el surco retroauricular y la región mastoidea. Se presentan 2 pacientes en los cuales se realizó el colgajo descrito con buenos resultados.
Los dos casos seleccionados con diagnóstico de carcinoma basocelular de la región auricular, entre los años 2009 y 2019 en una clínica privada de Concepción, en donde se consideró la necesidad de realizar este colgajo local para cobertura de los defectos secundario a la resección tumoral. Este trabajo cuenta con la autorización del comité de ética correspondiente y cautela la confidencialidad de los datos a través de los consentimientos informados de los pacientes.

\section{Casos Clínicos}

Los casos corresponden a varones de 55 y 63 años, ambos con diagnóstico de carcinoma basocelular de la región de la concha auricular. Uno de ellos se presentó en la oreja izquierda y el otro en la derecha. Luego de la resección con bordes libres por estudio inmediato de 
anatomía patológica, se procedió a diseñar el colgajo en isla de acuerdo al defecto a cubrir (Figura 1).

El tamaño del defecto fue entre $1,5 \mathrm{~cm}^{2}$ $2,0 \mathrm{~cm}^{2}$. El colgajo cutáneo retroauricular fue rotado de posterior a anterior manteniendo el puente de irrigación subcutánea a nivel del surco auricular. El tejido rotado fue suturado a los bordes del defecto anterior (Figura 2).

El defecto en la región posterior fue cubierto sin dificultad por avance cutáneo de la región retroauricular. El resultado de la cobertura y de la cicatriz de la región donante fueron consideradas aceptables por el paciente y el equipo quirúrgico. No hubo complicaciones inmediatas. El seguimiento a tres años no demuestra complicaciones ni recidivas tumorales.

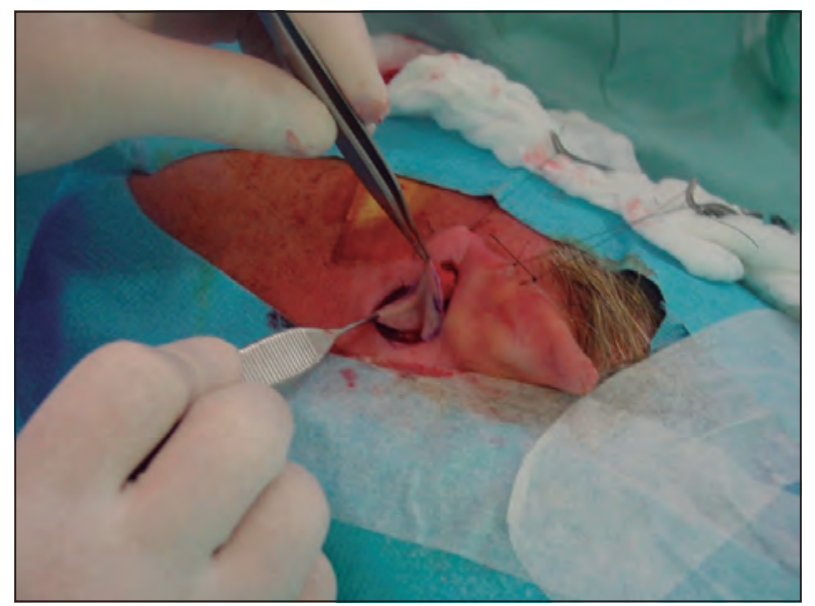

Figura 1. Colgajo flip-flop obtenido de región mastoidea.

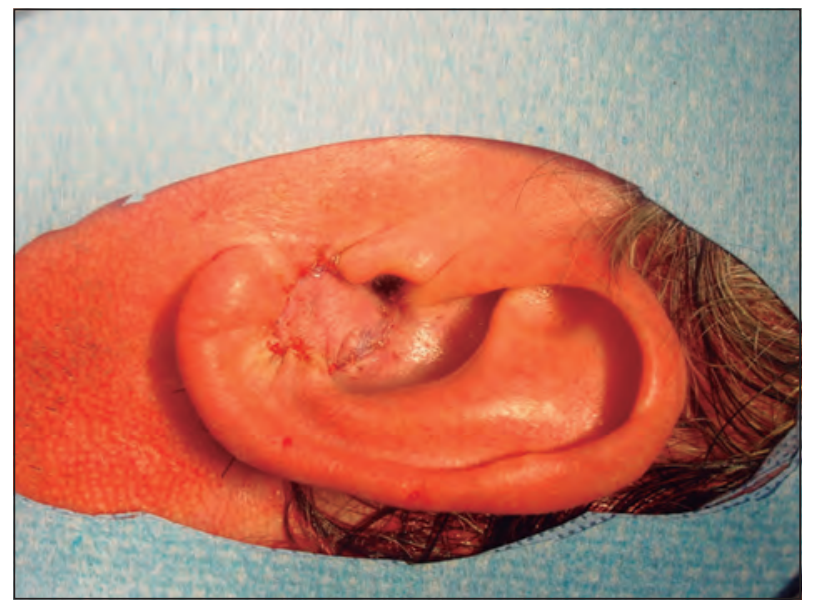

Figura 2. Rotación de colgajo flip-flop a concha auricular.

\section{Discusión}

Cuando hay un defecto de cobertura de la región conchal auricular, existe la posibilidad de realizar un cierre directo, cicatrización por segunda intención o injerto de piel total. Sin embargo, cuando estos defectos son más extensos o se sospecha ausencia de pericondrio o un terreno poco vascularizado, una alternativa versátil y segura es el colgajo cutáneo o fasciocutáneo en isla tipo flip-flop o giratorio retroauricular. Entre sus ventajas está que es fácil de diseñar y ejecutar, aporta su propia vascularización disminuyendo el riesgo de condritis e infecciones, tiene escasa retracción y baja posibilidad de isquemia; junto a su seguridad, textura y color adecuado proporciona un resultado funcional y estético favorable $e^{3,4}$.

\section{Conclusión}

El colgajo flip-flop o de puerta giratoria, proporciona una alternativa de reconstrucción de la concha auricular simple y confiable con resultados funcionales y estéticos satisfactorios, sobre todo en casos en que alternativas menores no estén indicadas.

\section{Bibliografía}

1. Turan A, Turkaslan T, Kul Z, Isler C, Ozsoy Z.

Reconstruction of the anterior surface of the ear using a postauricular pull-through neurovascular island flap. Ann Plast Surg 2006;56:609-613. Disponible en https://doi.org/10.1097/01.sap.0000203986.20454.26.

2. Masson JK. A simple island flap for reconstruction of concha-helix defects. Br J Plast Surg 1972;25:399403. Disponible en https://doi.org/10.1016/s00071226(72)80083-3.

3. Papadopoulos ON, Karypidis DK, Chrisostomidis CI, Konofaos PP, Frangoulis MB. One-stage reconstruction of the antihelix and concha using postauricular island flap. Clin Exp Dermatol 2008;33:647-650. Disponible en https://doi. org/10.1111/j.1365-2230.2008.02741.x.

4. Schonauer F, Vuppalapati G, Marlino S, Santorelli A, Canta L, Molea G. Versatility of the posterior auricular flap in partial ear reconstruction. Plast Reconstr Surg 2010;126:1213-1221. Disponible en https://doi.org/10.1097/PRS.0b013e3181ec1f03. 